\title{
Behavioral Evidence That Segregation and Representation Are Dissociable Hippocampal Functions
}

\author{
Štěpán Kubík ${ }^{1}$ and André A. Fenton ${ }^{1,2}$ \\ ${ }^{1}$ Laboratory of Neurophysiology of Memory, Institute of Physiology, Academy of Sciences of the Czech Republic, 14220 Prague 4, Czech Republic, and \\ ${ }^{2}$ Department of Physiology and Pharmacology, State University of New York, Brooklyn, New York 11203
}

\begin{abstract}
Hippocampal activity is thought to encode spatial representations in a distributed associative network. This idea predicts that partial hippocampal lesions would spare acquisition and impair retrieval of a place response as long as enough connections remained intact to encode associations. Water maze experiments supported the predictions, but the prediction of impaired retrieval was not supported when tetrodotoxin (TTX) was injected into one hippocampus and rats were tested in a place avoidance task on a rotating arena with shallow water. The rotation dissociated relevant distal stimuli from irrelevant self-motion stimuli. To explain the discrepancy, we hypothesized that the segregation of relevant and irrelevant stimuli and stimuli association into representations are distinct hippocampus-dependent operations, and whereas associative representation is more sensitive to disruption during retrieval than learning, stimulus segregation is more sensitive to disruption during learning than during retrieval. The following predictions were tested: (1) the TTX injection would spare learning but (2) impair retrieval of a place response in the water maze, which has a high associative representational demand but a low demand for segregation; (3) the injection would impair learning but (4) spare retrieval of place avoidance in the rotating arena filled with water, which has a high demand for stimulus segregation but a low associative representational demand. All four predictions were confirmed. The hypothesis also explains the pattern of sparing and impairment after the TTX injection in other place avoidance task variants, leading us to conclude that stimulus separation and association representation are dissociable functions of the hippocampus.
\end{abstract}

Key words: acquisition; retention; pattern separation; tetrodotoxin; reversible lesion; place avoidance

\section{Introduction}

Contemporary distributed memory hypotheses of hippocampus postulate that representational memory is encoded sparsely in the connections between cells (Marr, 1971; McNaughton and Morris, 1987; for review, see Lisman, 1999). Because a distributed subset of connections encodes memory, acquisition is spared by an incomplete lesion if sufficient connectivity survives. In contrast, if enough encoding synapses are destroyed, the same incomplete lesion will impair retrieval of memory that was encoded before the lesion. These predictions were confirmed when permanent partial lesions of the dorsal hippocampus spared acquisition and impaired retrieval of a place response in a water maze (Moser and Moser, 1998). Ordinarily, optimal behavior requires distinguishing relevant stimuli from irrelevant stimuli and associating the relevant stimuli in representational memory, but water maze paradigms emphasize representational memory by not dissociating relevant and irrelevant stimuli.

\footnotetext{
Received April 29, 2005; revised Aug. 18, 2005; accepted Aug. 24, 2005.

This work was supported by the 5th Framework Research and Technological Development Program of the European Commission (QLG3-CT-1999-00192), the J. S. McDonnell Foundation (98-38-CNS-QUA.05), and the Academy of Sciences of the (zech Republic (AV0Z5011922). We thank Eduard Kelemen, May-Britt Moser, and Ole Paulsen for useful discussions.

Correspondence should be addressed to Dr. André Fenton, State University of New York, Downstate Medical Center, 450 Clarkson Avenue, Brooklyn, NY 11203. E-mail: afenton@downstate.edu.

S. Kubík's present address: Institute of Neuroscience, 1254 University of Oregon, Eugene, OR 97403.

DOI:10.1523/JNEUROSCI.1707-05.2005

Copyright $\odot 2005$ Society for Neuroscience $\quad 0270-6474 / 05 / 259205-08 \$ 15.00 / 0$
}

The distributed memory predictions were not confirmed when tetrodotoxin (TTX) was injected into one dorsal hippocampus, and acquisition and retrieval of a place response was tested in a variety of hippocampus-dependent (Cimadevilla et al., 2000; Ježek et al., 2002) place avoidance tasks. Acquisition, retrieval, and consolidation of the place response were abolished when room and arena stimuli were continuously dissociated by rotating the arena (Cimadevilla et al., 2001; Wesierska et al., 2005). According to the distributed memory hypothesis, the minimum number of synapses needed to encode place avoidance memory was not available after the TTX injection. This account failed to explain why the same injections spared learning and retention when room and arena stimuli were not dissociated (Wesierska et al., 2005).

Rotating the arena dissociated room and arena stimuli and challenged the rat to segregate task-relevant stationary stimuli from irrelevant rotating stimuli in addition to associating the stationary cues with shock in a spatial representation. The distributed memory hypothesis can explain the water maze and the place avoidance data by assuming that stimulus segregation (orthogonalization) and distributed representational memory are separate, dissociable hippocampal functions. The hypothesis is represented schematically in Figure 1.

Place avoidance required avoiding one-sixth of the accessible space, so a less accurate place representation was needed than escaping to the small $(\sim 1 / 400$ th) target area in a water maze (Fig. 
A1
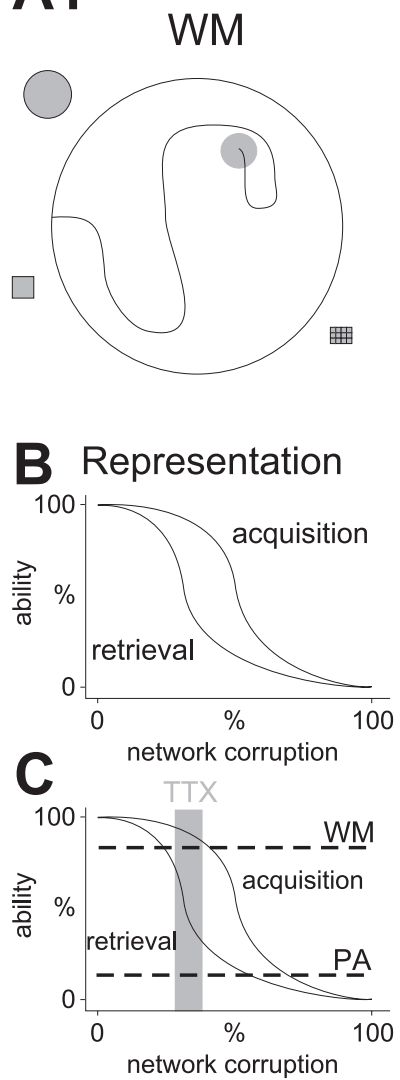

WM

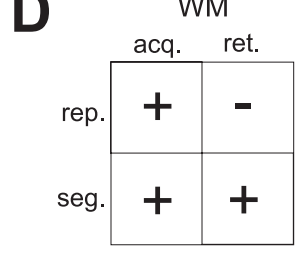

Perform. + -
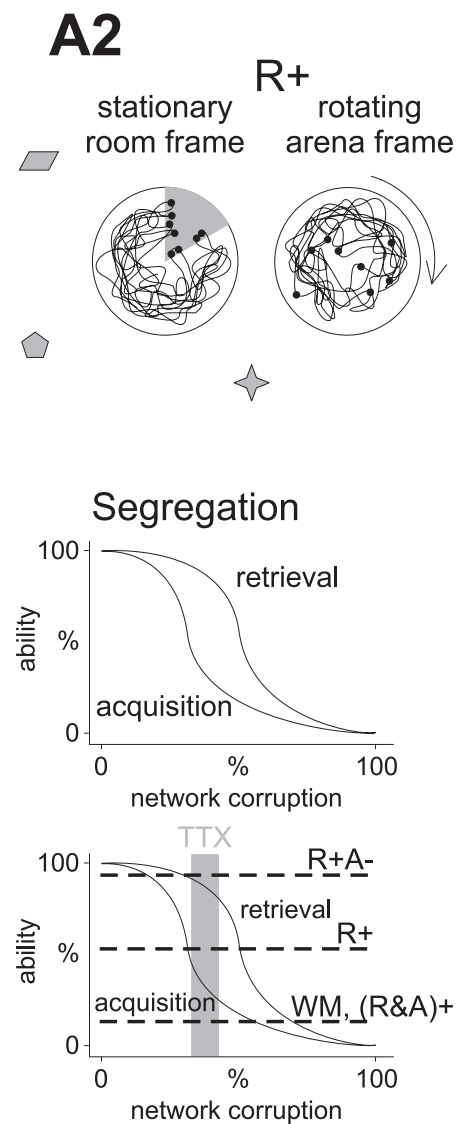

$\mathrm{R}+$

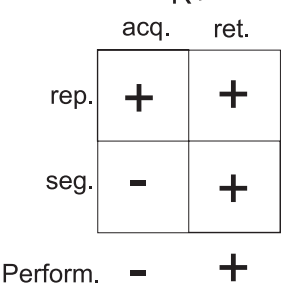

Figure 1. Schematic representation of the hypothesis that segregation and representation of stimuli are dissociable functions of the hippocampus. $A$, Water maze tasks require the rat to identify a hidden platform (gray circle), a relatively small part of a large space using extra-maze room stimuli, three of which are represented. The task and the track of a rat on a single trial before performance is optimal are depicted. Place avoidance tasks on the other hand require the rat to identify a relatively large portion (gray sector) of a smaller circular arena in which footshock occurs (small black circles). In the Room + task, the to-be-avoided place is defined by extra-maze room cues, three of which are represented. In the Room + task, the arena is filled with shallow water to hide arena-based olfactory stimuli, and the arena rotates to make selfmotion stimuli irrelevant for avoiding the stationary to-be-avoided place. As a result, although shocks only occur in a restricted part of the room, they occur throughout the arena and with no fixed relationship to self-motion stimuli. The task thus challenges the rat to segregate the relevant room stimuli from irrelevant self-motion stimuli. $\boldsymbol{B}$, Hypothesized relationships between the amount of corrupted connectivity in the hippocampus and the ability of the network to segregate and represent spatial relationships. It is hypothesized that segregation and representation are distinct functions of a distributed hippocampal network. The sparse and distributed nature of stored representations means that only a small portion of the hippocampus is required to acquire a representational memory but that retrieval of this memory is more sensitive to hippocampal corruption when all the encoding synapses are not available during memory recall. The opposite dissociations of acquisition and retrieval occur in the relationship between network corruption and ability to segregate. When segregation is compromised, acquiring the association between relevant stimuli and/or responses will be retarded by interference from irrelevant associations. Once the appropriate associations are formed, segregation is less critical, because only the relevant stimuli will have strengthened associations to appropriate responses. C, Injecting TTX into one hippocampus inactivates one hippocampus, producing what we estimate to be just under $50 \%$ network corruption. Water maze tasks require a

$1 A)$. Water in the water maze hides local stimuli, and because distal and self-motion stimuli are mutually stable, less segregation is required compared with avoiding a stationary location while walking on a rotating arena filled with water (Fig. $1 \mathrm{~A}$ ). As explained above, representational capacity is more sensitive to dysfunction during retrieval than acquisition (Fig. $1 B$ ). In contrast, segregation capacity is more sensitive to dysfunction during acquisition than retrieval (Fig. $1 B$ ). This is because both appropriate and inappropriate stimulus-stimulus and stimulus-response associations can form during impaired stimulus segregation, but segregation is less crucial when only the appropriate associations already exist for retrieval. The present experiments tested the predictions that the unilateral TTX injection would spare acquiring and impair retrieving a place response in the water maze (Fig. 1C,D) but impair acquiring and spare retrieving a place response on a rotating arena filled with shallow water (Fig. $1 C, D)$.

\section{Materials and Methods Subjects}

Thirty-three adult male Long-Evans rats from the Institute of Physiology breeding colony were used. They were housed at $21^{\circ} \mathrm{C}$. All manipulations were performed in the light phase of a $12 \mathrm{~h} \mathrm{light/dark} \mathrm{cycle.} \mathrm{All} \mathrm{experi-}$ ments were performed in accordance with the Animal Protection Act of the Czech Republic, National Institutes of Health guidelines, and the directive of the European Communities Council (86/609/EEC).

\section{Surgery}

The methods for surgical implantation of guide cannulas and the infusion of TTX have been described previously in detail (Fenton and Bureš, 1993; Fenton et al., 1995; Cimadevilla et al., 2000, 2001; Klement et al., 2005; Wesierska et al., 2005). Rats were anesthetized ( $50 \mathrm{mg} / \mathrm{kg} \mathrm{Nembu-}$ tal; Sigma, St. Louis, MO), and the skull was exposed. Two holes were drilled to aim two 22 ga 10 -mm-long stainless-steel guide cannulas at the dorsal hippocampi (relative to bregma: posterior, 3.5 ; lateral, \pm 2.6 ; ventral, 2.0). Two anchoring bolts were implanted in slits drilled into the skull, then cemented to the bone and the cannulas with dental acrylic. Lubricated stainless-steel stylets were inserted into the cannulas to prevent occlusion. Each rat was also implanted with a subcutaneous lowimpedance electrode. The electrode was made by bending a 25 ga hypodermic needle into a $\mathrm{U}$-shaped pin, piercing the skin between the shoulders, and soldering a screw to the sharp end. The screw prevented the electrode from slipping out and provided purchase for an alligator clip that could connect the electrode to a wire that delivered shock. The rats had $8-16 \mathrm{~d}$ to recover from the surgery.

\section{Intrahippocampal injection}

Tygon tubing (Small Parts, Miami Lakes, FL) connected a 30 ga injection needle to a $10 \mu \mathrm{l}$ Hamilton syringe. The rat was restrained by hand, and

$\leftarrow$

relatively fine spatial representation, and place avoidance tasks $(\mathrm{PA})$ require a relatively coarse place representation. The effect of the ITX injection has been determined on three variants of place avoidance, all of which have the same representational demand. Water maze tasks require relatively little stimulus segregation. The Room and Arena $+[(R \& A)+]$ place avoidance task, in which the arena does not rotate, is similar to the water maze in that there is little demand for stimulus segregation. The segregation demand is higher for the Room + task, in which shallow water hides arena stimuli, and rotation dissociates room stimuli from selfmotion stimuli. Room + Arena - avoidance $(R+A-)$ has an even greater demand for stimulus segregation, because arena rotation dissociates room stimuli from olfactory arena stimuli and self-motion stimuli. The level of network corruption by TTX may leave sufficient network capacity for segregation or representation for one task but not another. $\boldsymbol{D}$, A contingency table derived from ( summarizes the predictions of the hypothesis. There will be opposite dissociations of acquisition (acq.) and retrieval (ret.) in the water maze and Room + avoidance tasks. These predictions are tested in the current study. WM, Water maze. $R+$, Room + task; rep., representation; seg., segregation; Perform., performance. 
the stylets were removed. The injection needle was inserted through one of the guide cannulas until a stopper positioned the needle tip $3.5 \mathrm{~mm}$ ventral to bregma. One microliter of saline or TTX solution $(5 \mathrm{ng} / \mu \mathrm{l}$ saline; Sigma) was injected continuously over a minute. The injection needle was left in place for another minute and then slowly retracted. The $5 \mathrm{ng}$ dose of TTX was determined to block neural transmission in an $\sim 1$ $\mathrm{mm}$ sphere for $\sim 5 \mathrm{~h}$ (Zhuravin and Bureš, 1991). The rats were injected $1 \mathrm{~h}$ before training. The injections were left/right counterbalanced across animals, but a rat was always injected in the same hippocampus. One bilateral injection of TTX was administered a few days before the behavioral training started to habituate the rats to the injection procedure and to the TTX-induced state before training. No signs of ataxia were observed before training, suggesting the TTX did not diffuse to the thalamus.

\section{Histology}

After completion of the experiments, the rats were anesthetized with an overdose of thiopental $(100 \mathrm{mg} / \mathrm{kg})$ and perfused transcardially with saline and then $10 \%$ formalin. The brains were removed, sectioned, and stained with cresyl violet, and the injection sites were verified to be in the dorsal hippocampus within $0.5 \mathrm{~mm}$ of the target (see Fig. 2C). Three rats with necrosis or lesions extending beyond the hippocampus were excluded from analysis.

\section{Water maze apparatus}

A $1.83-\mathrm{m}$-diameter circular pool filled with 40 -cm-deep $21-22^{\circ} \mathrm{C}$ water was used. The pool was centered in a $3.8 \times 4.1 \mathrm{~m}$ room with a rich set of distal visual cues. A circular, 10-cm-diameter, clear Perspex escape platform was submerged $1 \mathrm{~cm}$ below the water in the center of an arbitrarily designated quadrant. The rat's position was tracked and analyzed by a computer-based video tracking system (iTrack and TrackAnalysis; BioSignal Group, Brooklyn, NY).

\section{Water maze paradigm}

Rats were released facing the wall at one of the four cardinal compass directions (North, West, South, and East). If a rat did not escape onto the platform in $60 \mathrm{~s}$, it was guided there by hand and left to rest for 15-30 s. The rats were released from the center facing away from the target quadrant on probe swims. Twelve rats were used in the water maze experiments.

\section{Water maze data analyses}

The average daily escape latencies were analyzed by two-way ANOVA (groups-by-days with repeated measures on days). Swimming speed was also analyzed. The percentage of time spent in the target quadrant during the first $30 \mathrm{~s}$ of a probe swim was used to measure retention of a place response. It was analyzed by one-way ANOVA for the effect of groups and by one-sample $t$ test for the difference from chance. Performance on probe swims given on different days was compared by two-way ANOVA with repeated measures on days. Newman-Keuls post hoc comparisons were performed when appropriate. Significance was accepted at $p<0.05$. Means \pm SEM are reported.

\section{Room + place avoidance apparatus}

The place avoidance system (Bio-Signal Group) was adapted from that used in previous studies (Bureš et al., 1997; Fenton et al., 1998; Cimadevilla et al., 2000, 2001). The arena was a uniform metal disk, $82 \mathrm{~cm}$ in diameter, with a 5-cm-high wall. It was filled with a 1-cm-deep layer of water and mounted on a $76-\mathrm{cm}$-high turntable that could be rotated at 1 rpm by an electric motor. The arena was centered in a room with multiple distal landmarks. The water was used to obscure task-irrelevant olfactory cues and to make droppings unstable. The rat wore a latex harness that held infrared light-emitting diodes (LEDs) between the shoulders. The LEDs were powered through a cable. The cable also carried the shock current, which was connected to the implanted electrode by an alligator clip. Shock current $(50 \mathrm{~Hz}, 0.5 \mathrm{~s}, 0.5-0.7 \mathrm{~mA})$ from a constant-current source was delivered through the implanted low-impedance $(\sim 100 \Omega)$ shock electrode and across the high-impedance $(\sim 100 \mathrm{k} \Omega)$ contact be- tween the rat's wet parts of the body and the grounded arena. The shock current was monitored by the experimenter. A computer-based tracking system (iTrack; Bio-Signal Group) analyzed the signal from an overhead television camera and recorded the position of the LEDs on the rat 25 or 50 times per second. The position time series were stored for off-line analyses (TrackAnalysis; Bio-Signal Group). Two experimental rooms were used in this experiment. The downstairs laboratory was $2.3 \times 3.0 \mathrm{~m}$, and the upstairs laboratory was $2.3 \times 2.3 \mathrm{~m}$. The two rooms were visually distinct.

\section{Room + place avoidance pretraining}

Twenty-one rats were used in this experiment, three of which were excluded from the analysis based on the histological findings. The rats were food deprived to $85 \%$ of their free-feeding weight. Then they were habituated to the arena in the downstairs laboratory during three sessions with no shocks. They were trained to forage for cocoa puffs that were dropped into the arena from an overhead feeder every $20 \mathrm{~s}$. The cocoa puffs floated and did not dissolve before being eaten. The rats foraged almost continuously by the third session. The arena was stable in the first session, and three rats were on the arena at a time. The arena rotated continuously at $1 \mathrm{rpm}$ clockwise in the second and third sessions. Only one rat was on the rotating arena at a time in the third session.

\section{Place avoidance paradigm}

A rat was placed on the arena across from the to-be-avoided sector facing away from the arena center. The arena started to rotate at $1 \mathrm{rpm}$ clockwise, and a cocoa puff was dropped into the arena every $20 \mathrm{~s}$. The Room + task required the rat to avoid a $60^{\circ}$ sector that was defined in the coordinates of the room while the arena rotated continuously. Whenever the rat entered the to-be avoided sector for $>0.5 \mathrm{~s}$, the tracking system delivered a mild constant-current footshock and counted an entrance. The current amplitude for each rat was adjusted in the first session to be the minimum necessary to elicit flinch and escape responses (Cimadevilla et al., 2000) and was not changed afterward. If the rat did not leave the sector, additional shocks were given every $1.5 \mathrm{~s}$ until the rat left the sector, but no more entrances (E) were counted until the rat left the sector for $>0.5 \mathrm{~s}$. Each session lasted $20 \mathrm{~min}$, and the total number of entrances per session was used to measure avoidance within the session. The latency to enter the to-be-avoided sector for the first time in a session (T1) was used to measure the between-session retention of avoidance memory.

\section{Room + avoidance data analyses}

Performance across days was analyzed by two-way ANOVA (groups-bydays) with repeated measures on days. The effect of TTX and saline injections was tested in different groups of rats. Each rat was trained on both a novel and a familiar place avoidance. To compare performance in the novel and familiar conditions, the average performance across days in each condition was compared by two-way ANOVA (groups-bycondition) with repeated measures on condition. Within-subject comparisons were done by paired $t$ tests and one-way ANOVA. NewmanKeuls post hoc comparisons were performed when appropriate. Significance was accepted at $p<0.05$. Means \pm SEM are reported.

\section{Experimental design}

\section{Experiment 1: retention of water maze escape}

Days 1-5 (initial training). The rats were trained to escape to the platform in the Northeast quadrant on eight daily training trials with intertrial interval of $15 \mathrm{~min}$. The rats were not injected during the first $4 \mathrm{~d}$. On day 5 , each rat received a saline injection into one hippocampus, and $1 \mathrm{~h}$ later, it was given a $60 \mathrm{~s}$ probe swim with the escape platform removed. The rats were then trained the same way as on the previous $4 \mathrm{~d}$. The probe swim on day 5 was done to verify that the rats had good $24 \mathrm{~h}$ retention before testing the effect of the TTX injection.

Day 6 (injected probe swim). The rats were divided into two groups. Six rats received a TTX injection, and six rats received a saline injection $1 \mathrm{~h}$ before a 60 s probe swim. 
Experiment 2: acquisition of water maze escape

Days 1-5 (injected training). Eleven of the rats from experiment 1 were used because one rat lost its implant. The rats were injected with TTX or saline so that three TTX- and three saline-injected rats from the previous phase were in the TTX group and three TTX- and two saline-injected rats were in the saline group. The daily training was identical to that in experiment 1 . Training started $1 \mathrm{~h}$ after the injections. The probe trial at the start of day 5 tested whether the rats had acquired a place response under TTX blockade by testing whether the response was retained across $24 \mathrm{~h}$.

Day 6 (injected probe swim). The rats were injected as on previous days, and $1 \mathrm{~h}$ later, a $60 \mathrm{~s}$ probe swim was given. This second probe trial tested again whether the rats had acquired a place response under TTX inactivation.

Day 7 (uninjected probe swim). The rats were given a $60 \mathrm{~s}$ probe swim with no injections to determine whether retrieval of the place response that was acquired under the influence of the injections was state dependent.

\section{Experiment 3: acquisition of Room + avoidance}

The present experiment was designed after a pilot experiment revealed that Room + avoidance was extremely poor in naive rats injected in one hippocampus with TTX. Because it was possible that the poor performance was because the injection impaired some procedural aspect of the task, we first trained rats to do Room + avoidance without the injections to ensure they had learned the cognitive and procedural features of the task. We then tested whether they could learn a new Room + place avoidance in a novel environment after the TTX injections.

Days 1-4 (initial uninjected training). Eleven experimentally naive rats were trained to avoid a place in the stationary room while the arena filled with shallow water rotated in the Room + avoidance task in the downstairs laboratory.

Days 9-14 (injected novel training). Each day, TTX $(n=6)$ or saline $(n=5)$ was injected into one hippocampus, and $1 \mathrm{~h}$ later, the rat was trained to do Room + avoidance in the novel, upstairs laboratory.

\section{Experiment 4a: retention of Room + avoidance}

Injected relearning. The rats from experiment 3 were used. On days 1517 , they were injected in the same hippocampus with the same solution as before and trained for $3 \mathrm{~d}$ on the same place avoidance in the familiar Room + avoidance task that was learned in initial uninjected training.

\section{Experiment 4b: retention of Room + avoidance: control for} repeated injections

A new group of seven rats received the same uninjected initial training as the rats in experiment 3 and $4 \mathrm{a}$. These new rats were neither trained nor injected during the time corresponding to the new learning (days 9-14) in experiment 3. They were injected with TTX on days 15-17 and retrained in the familiar avoidance. Thus rats in this control group ("Wait") had the identical treatment to the TTX-injected rats in experiment $4 \mathrm{a}$ with the exception that the Wait animals were not trained in the novel environment and they were injected for the first time in the familiar relearning sessions. Performance in the Wait and TTX-injected group from experiments 3 and $4 \mathrm{a}$ were compared.

\section{Results}

\section{Experiment 1: retention of water maze escape}

The hypothesis that hippocampus-dependent stimulus segregation and associative representation are dissociable predicts the unilateral TTX injection will impair retention of a place response in the water maze if the response was learned before the injection. This experiment tested the effect of the TTX injection on retention of water maze escape.

\section{Days 1-5 (initial training)}

Rats in both groups learned to escape to the platform equally well during the $5 \mathrm{~d}$ (Fig. 2A1). Although the latency to escape was asymptotic by day 4 , we found no preference for the target quadrant in the day 5 probe swim (data not shown). The groups did not differ $\left(F_{(1,10)}=0.71 ; p>0.4\right)$. Neither the rats that would be
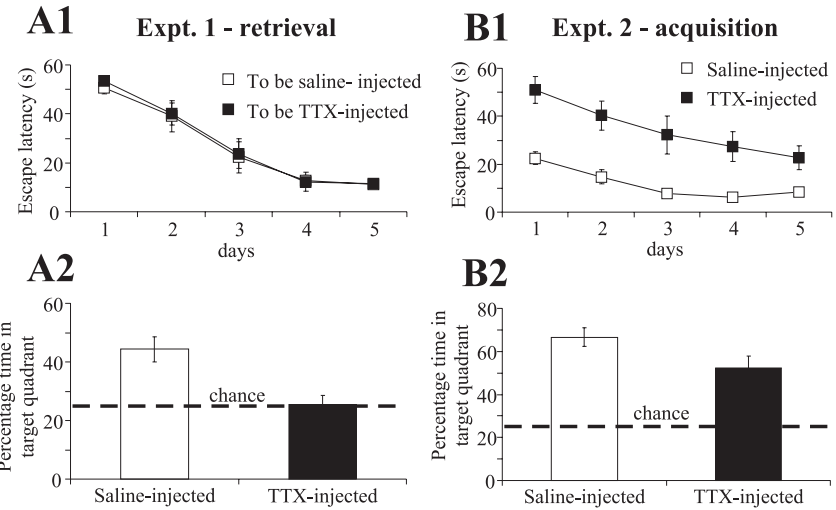

B2
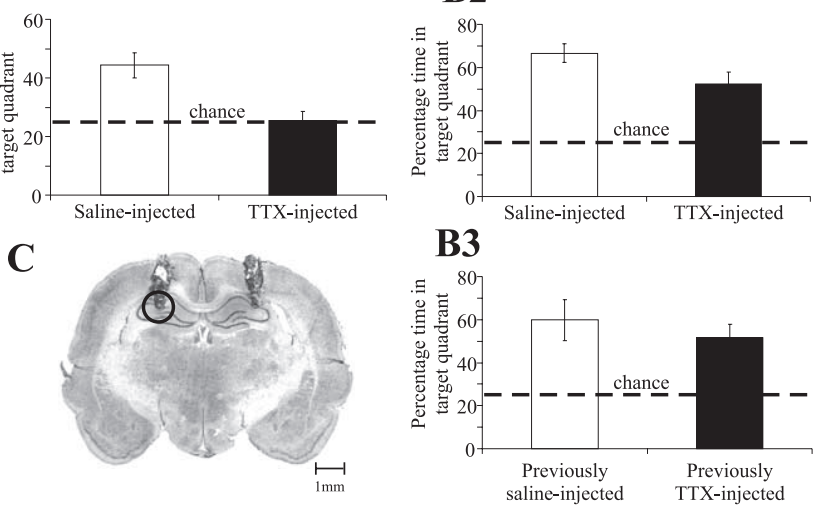

Figure 2. The TTX injection impaired retrieval of water maze escape learned before the injection (experiment 1) but spared learning a new escape (experiment 2). $\boldsymbol{A} \mathbf{1}$, The place response during initial training was similar in the two groups $\left(F_{(1,10)}=0.04 ; p>0.8\right)$ and improved significantly across days $\left(F_{(4,40)}=58.8 ; p<10^{-6} ;\right.$ day $1>$ day $2>$ day $3>$ day $4=$ day 5). $\mathbf{A 2}$, TTX injection before the probe swim abolished the place response. The groups were different $\left(F_{(1,10)}=10.7 ; p<0.01\right)$. The saline-injected rats preferred the target quadrant $\left(t_{(5)}=4.48 ; p<0.01\right)$, and the TTX-injected rats did not $\left(t_{(5)}=0.19 ; p>0.8\right)$. B1, Escape latencies in the novel water maze decreased across days $\left(F_{(4,36)}=14.8 ; p<10^{-6}\right.$; day $1>$ day $2>$ day $3=$ day $4=$ day 5$)$, but the TTX-injected rats were consistently slower than the saline-injected rats $\left(F_{(1,9)}=11.9 ; p<0.01\right)$. B2, Both groups had similar $\left(F_{(1,9)}=3.45 ; p>\right.$ 0.09 ) localized place responses on the day 6 probe swim (saline, $t_{(4)}=9.84, p<0.001 ; \mathrm{TTX}, t_{(5)}=$ $5.08, p<0.005)$. B3, The two groups also had similar place responses $\left(F_{(1,9)}=0.44 ; p>0.5\right)$ on the subsequent uninjected probe swim (saline, $t_{(4)}=3.64, p<0.05 ; \operatorname{TTX}_{1} t_{(5)}=4.17, p<$ 0.01). C, Photomicrograph depicting the injection cannula placement in a rat. The circle on the left shows the expected region of effective TTX diffusion. Expt., Experiment. Error bars represent SEM.

injected with $\operatorname{TTX}\left(t_{(5)}=0.82 ; p>0.4\right)$ nor the to-be-injected saline rats $\left(t_{(5)}=1.54 ; p>0.1\right)$ preferred the target quadrant, so the training was continued on day 5 . This result indicates that it requires more training for rats to learn to persistently search at the target location than for them to learn to escape.

Day 6 (injected probe swim)

The TTX injection impaired retention of the place response in the water maze (Fig. 2A2), similar to the effects of bilateral partial permanent lesions or temporarily inactivating muscimol injections (Moser and Moser, 1998). The saline-injected rats preferentially searched the target quadrant $\left(t_{(5)}=4.48 ; p<0.01\right)$, but the TTX-injected rats did not $\left(t_{(5)}=0.19 ; p>0.8\right)$.

\section{Experiment 2: acquisition of water maze escape}

The hypothesis that stimulus segregation and associative representation are dissociable hippocampal functions predicted the unilateral TTX injection would spare learning a place response in the water maze. This experiment tested whether the TTX injection impaired acquiring a place response in the water maze.

A different water maze from the one used in experiment 1 was created by adding a white noise source and using a black curtain with multiple visual cues to surround the pool.

Days 1-5 (injected training)

The rats learned a place response. Escape latencies decreased to asymptotic values by day 3 , but the TTX-injected rats took signif- 
icantly longer to escape to the platform than the saline-injected rats (Fig. 2 B1). Despite this apparent learning impairment, both groups preferred the target quadrant equally well during the pretraining probe swim on day 5 (Fig. 2B2). We investigated whether this discrepancy could be caused by a difference in swimming speed but found that the TTX-injected rats actually swam faster $\left(F_{(1,9)}=12.6 ; p<0.01\right)$ (data not shown). These results are consistent with our observation that the TTX-injected animals appeared to have procedural difficulties. They were often observed to just miss the escape platform, passing in close vicinity, whereas the saline-injected animals usually found the platform once they were close to it. The increased speed seemed to reflect an impaired ability to execute a single behavior at any moment, which was compensated for by hyperlocomotion. This impression is based in part on the observation that approximately onehalf of the TTX-injected rats did not readily follow the experimenter's hand that tried to guide them to the platform after the $60 \mathrm{~s}$ trial had elapsed during the initial blocks of training under TTX. This behavior was observed during the initial training trials of experiment 1 but in none of the saline-injected rats in the current experiment. It seemed to us that the discrepancy between the impaired escape latencies and the unimpaired place response in the TTX-injected rats indicated an impairment in the process of selecting appropriate escape behaviors rather than an impairment in place navigation itself.

\section{Day 6 (injected probe swim)}

All rats preferred the target quadrant (Fig. 2 B2), demonstrating that in accordance with previous reports (Fenton and Bureš, 1993, 1994; Fenton et al., 1995), the unilateral injection of TTX does not prevent learning or expressing a place response in the water maze. Both groups performed well in the probe swims on days 5 and 6 (groups, $F_{(1,9)}=3.43, p>0.09$; probes, $F_{(1,9)}=1.69$, $p>0.2$; interaction, $\left.F_{(1,9)}=0.12, p>0.7\right)$. Because performance was similarly good on days 5 and 6 , the lack of a group difference cannot be attributed to a weak but improving place response.

Day 7 (uninjected probe swim)

All rats preferred the target quadrant (Fig. 2 B3), demonstrating that the learned place response was not specific to the state induced by the TTX injection. Presumably a generally accessible place response was formed under the TTX inactivation.

\section{Experiment 3: acquisition of Room + avoidance}

The hypothesis that stimulus segregation and representational memory are dissociable hippocampal functions predicts that learning Room + avoidance will be impaired by the TTX injection, because distal room stimuli and self-motion cues must be segregated to selectively associate a room location with shock. This experiment tested whether the TTX injection would impair Room + avoidance learning.

\section{Initial uninjected training}

All rats learned Room + avoidance during $4 \mathrm{~d}$ (Fig. 3A). Grouping the data according to the treatment in the subsequent injected novel training phase confirmed that the groups were similar before the injections.

\section{Injected novel training}

The saline-injected rats but not the TTX-injected rats learned the Room + place avoidance in the new environment (Fig. $3 A$ ).

\section{Experiment 4a: retention of Room + avoidance}

The hypothesis that hippocampus-dependent stimulus segregation and associative representation are dissociable functions pre-
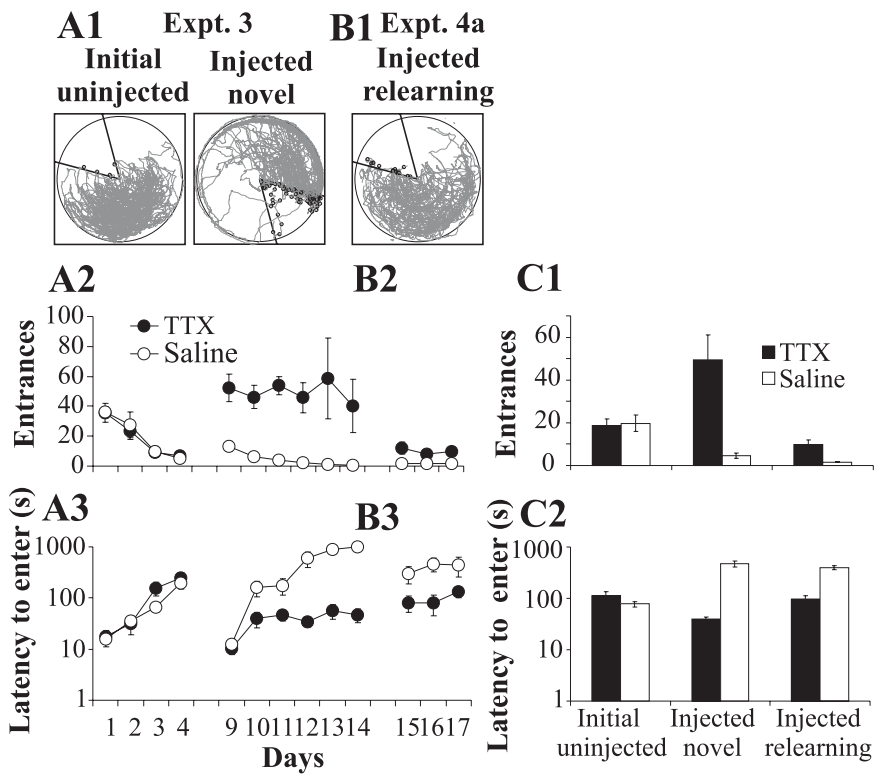

Figure 3. Experiments 3 and $4 a$. $\boldsymbol{A}$, The rats learned a Room + place avoidance during the initial uninjected training phase (days 1-4). There was a significant effect of days on both entrances and latency to first enter $\left(E, F_{(3,27)}=22.1, p<10^{-6}\right.$, day $1>$ day $2>$ day $3=$ day $4 ; \mathrm{T} 1, F_{(3,27)}=11.7, p<10^{-4}$, day $1=$ day $2<$ day $3<$ day 4$)$. Performance was similar when the rats were grouped according to whether they would be injected with saline or TTX ( $E$, $\left.F_{(1,9)}=0.04, p>0.8 ; \mathrm{T} 1, F_{(1,9)}=1.72, p>0.2\right)$. Compared with saline, injecting TTX on day 9-14 prevented learning a new Room + place avoidance in a different room $\left(E, F_{(1,9)}=10.2\right.$, $\left.p<0.02 ; \mathrm{T} 1, F_{(1,9)}=38.8, p<0.001\right)$. The effect of days $\left(\mathrm{E}_{,} F_{(5,45)}=0.50, p>0.7 ; \mathrm{T} 1, F_{(5,45)}=\right.$ $\left.20.7, p<10^{-6}\right)$ and the interaction were significant on entrance latencies $\left(E, F_{(5,45)}=0.36\right.$, $\left.p>0.8 ; \mathrm{T} 1, F_{(5,45)}=18.7, p<10^{-6}\right)$. The saline-injected rats made many fewer entrances than the TTX-injected rats from the first day, and they also took much longer to enter the to-be-avoided place for the first time on days 4-6. B. Experiment 4a. In contrast, both the saline- and TTX-injected rats performed the initially learned Room + place avoidance on days $15-17$. The saline-injected rats were better $\left(E, F_{(1,9)}=10.1, p<0.02 ; \mathrm{T} 1, F_{(1,9)}=50.4, p<\right.$ $\left.10^{-4}\right)$. Neither group improved across days $\left(E_{,} F_{(2,18)}=0.98, p>0.3 ; \mathrm{T} 1, F_{(2,18)}=0.33, p>\right.$ $0.7)$, and there was no interaction $\left(\mathrm{E}_{,} F_{(2,18)}=1.00, p>0.3 ; \mathrm{T} 1, F_{(2,18)}=0.19, p>0.8\right) . A 1$, $B 1$, Room-frame tracks of a representative TTX-injected rat on the last day of each phase in experiment $3(\boldsymbol{A} 1)$ and experiment $4 \mathrm{a}(\boldsymbol{B} 1)$. The small circles indicate locations at which the rat received shocks, and the black lines represent the to-be-avoided sector. $\mathbf{A 2}, \boldsymbol{A} \mathbf{3}, \boldsymbol{B} \mathbf{2}, \boldsymbol{B} \mathbf{3}$, The number of entrances $(\boldsymbol{A} 2)$ and the latency to first enter $(\boldsymbol{A} \mathbf{3})$ in experiment 3 and experiment $4 \mathrm{a}$ (B2, B3). C, Across-phase comparisons of the number of entrances (C1) and the latency to first enter $(\boldsymbol{C})$ indicate the TTX-injected rats were severely impaired only when they had to learn a new place avoidance in the novel environment. The analysis indicated that the saline-injected rats avoided better $\left(E, F_{(1,9)}=24.1, p<0.001 ; \mathrm{T} 1, F_{(1,9)}=105, p<10^{-5}\right)$, and there were significant effects of the experimental phase $\left(\mathrm{E}_{,} \mathrm{F}_{(2,18)}=4.75, p<0.05 ; \mathrm{T1}, F_{(2,18)}=11.4, p<\right.$ $0.001)$ and the interaction $\left(E_{,} F_{(2,18)}=6.06, p<0.01 ; \mathrm{T1}, F_{(2,18)}=20.1, p<10^{-4}\right)$. The TTX-injected rats made more entrances in the novel training phase than in both the initial training and the relearning phases. The saline-injected controls took longer to first enter in the novel training and the relearning phases. Expt., Experiment. Error bars represent SEM .

dicted that the TTX injection would impair learning Room+ avoidance but spare retention of a familiar Room + avoidance. Experiment 2 by Wesierska et al. (2005) trained rats to asymptotic performance of Room + avoidance without injections. Afterward, injecting TTX into one hippocampus did not impair the place response, confirming the prediction. The rats from experiment 3 were used to test the prediction in a second way. Experiment 4 a tested whether the TTX injection would impair retention of the Room + avoidance that was learned in the initial uninjected training phase of experiment 3 , although learning in the novel environment was impaired in the same rats.

\section{Injected relearning}

Both the saline- and the TTX-injected rats performed well, although the saline-injected rats performed better (Fig. 3B). Al- 


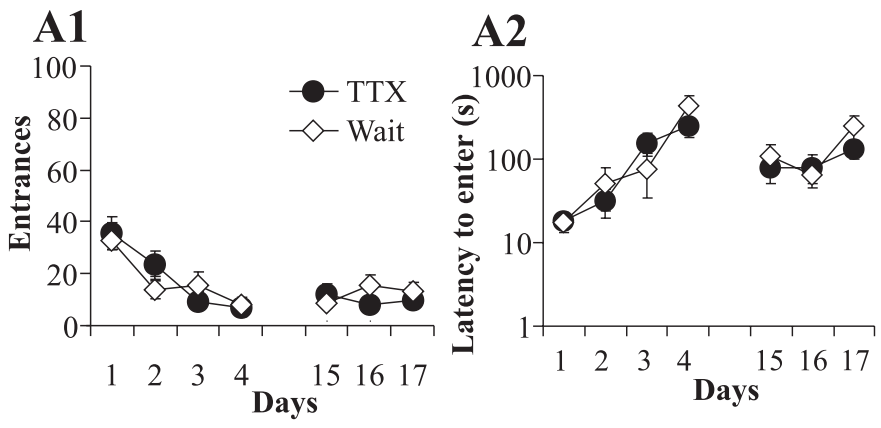

Figure 4. Experiments $4 a$ and $4 b$. The data from experiment $4 a$ are reproduced for comparison with those from experiment $4 \mathrm{~b}$. The groups were similar. They learned the Room + place avoidance during the initial uninjected training phase as measured by entrances $(\boldsymbol{A} \boldsymbol{1})$ and latency to first enter $(A 2)\left(E, F_{(1,11)}=0.07, p>0.7 ; \mathrm{T} 1, F_{(1,11)}=0.29, p>0.6\right)$. There was a significant effect of days $\left(E, F_{(3,33)}=25.2, p<10^{-6} ; \mathrm{T1}, F_{(3,33)}=12.1, p<10^{-4}\right)$ and no interaction $\left(\mathrm{E}_{1} F_{(3,33)}=2.09, p>0.1 ; \mathrm{T} 1, F_{(3,33)}=1.88, p>0.1\right)$. The rats entered the most on day 1 and improved between days 2 and 4 . They also took longer to enter the shock area for the first time on day 4. Both groups of rats also showed good retention of the place response after TTX injection in the relearning phase. There was no effect of groups $\left(E_{,} F_{(1,11)}=0.48, p>0.5\right.$; $\left.\mathrm{T} 1, F_{(1,11)}=1.15, p>0.3\right)$, days $\left(\mathrm{E}, F_{(2,22)}=0.14, p>0.8 ; \mathrm{T} 1, F_{(2,22)}=2.74, p>0.08\right)$, or interaction $\left(E_{,} F_{(2,22)}=2.64, p>0.09 ; T 1, F_{(2,22)}=0.71, p>0.5\right)$. Error bars represent SEM

though performance of the TTX-injected rats was worse than the saline-injected rats, it was still far better than during training in the new room under TTX (Fig. 3C1,C2). This indicates significant retention of the familiar place avoidance, which can also be measured by calculating savings as the difference between performance in the novel training and relearning sessions. The TTXinjected rats entered substantially fewer times in the first relearning session compared with the first session in the novel training $\left(F_{(1,5)}=8.42 ; p<0.05\right)$.

\section{Experiment 4b: retention of Room + avoidance: control for repeated injections}

The hypothesis that stimulus segregation and associative representation are dissociable hippocampal functions correctly predicted the results of experiments 3 and $4 a$, that the TTX injection would impair new avoidance learning more than retention in the relearning test. There is, however, an alternative explanation of these data, that because of the repeated TTX injections, the effectiveness of the neural activity block was reduced in the relearning test (experiment 4a) compared with in the new learning test (experiment 3). Experiment $4 \mathrm{~b}$ was performed to examine this possibility.

\section{Initial uninjected training}

Not surprisingly, initial learning of the rats in the Wait group was not different from the to-be TTX-injected rats in experiment 3 (Fig. 4A1,A2).

\section{Injected relearning}

Importantly, performance of the Wait group was indistinguishable from the performance of the TTX-injected rats during relearning of the familiar avoidance (Fig. 4A1,A2). Neither group improved indicating performance was asymptotic.

\section{Discussion}

\section{Dissociated acquisition and retrieval}

The reversible lesions by TTX permitted within-subject comparisons and did not allow spared function to be attributed to lesioninduced reorganization of brain circuitry (Bureš and Burešova, 1990; Klement et al., 2005). The TTX injections blocked neural activity for at least $5 \mathrm{~h}$ (Harlan et al., 1983; Zhuravin and Bureš, 1991).

In accord with Moser and Moser (1998), the present study found that injecting TTX into one hippocampus impaired retrieval of a place response in the water maze that was learned before the injection (Fig. $2 \mathrm{~A}$ ), but the injection did not prevent learning a place response in a novel water maze (Fig. $2 B$ ). The same injection prevented learning a Room + avoidance in a novel environment (Fig. $3 A, C$ ) but did not prevent retrieval of an avoidance that was learned before the injections (Figs. $3 B, C, 4$ ) (Wesierska et al., 2005). Abolished learning of a new Room+ avoidance was probably not because of difficulties learning task procedures, because the rats learned the task in a different environment before the injections and demonstrated subsequent retrieval of the familiar avoidance. Preserved retrieval could not be attributed to fast relearning for several reasons. First, performance during retraining did not change across days. Within a session, there was a measurable improvement of performance across 5 min intervals, and this pattern of improvement was similar on each of the 3 relearning days (data not shown). Second, the saline-injected rats continuously improved over at least three sessions in a novel environment, even if they were already familiar with the place avoidance procedure. Most importantly, if one assumes that the TTX-injected rats had no accessible memory for the to-be-avoided place at the start of the relearning phase, it is difficult to explain why the rats did the familiar Room + avoidance when they could not learn the avoidance in a new environment. Finally, the TTX-injected rats had substantial savings in the first relearning trial compared with the first trial in the novel environment. We conclude that place avoidance acquired during initial training was retrieved in the relearning trials.

The pattern of relatively spared place learning but impaired retrieval in the water maze and impaired place learning but relatively spared retrieval of a place response in the place avoidance task confirmed the predictions of the hypothesis diagrammed in Figure 1. These data suggest that stimulus segregation and associative representation are dissociable properties of hippocampus.

\section{Pattern separation and pattern completion}

Pattern separation (orthogonalization) was hypothesized to precede associative operations in distributed hippocampal processing of neural representations (Marr, 1971; McNaughton and Nadel, 1989; O'Reilly and McClelland, 1994; Shapiro and Olton, 1994; Rolls and Treves, 1998; Kesner et al., 2000). Within the viewpoint of serial processing along the trisynaptic pathway, pattern separation is assigned to the dentate gyrus, whereas pattern completion via autoassociation is ascribed to CA3 (McNaughton and Morris, 1987; McClelland et al., 1995). However, the trisynaptic loop may not be the dominant processing pathway through hippocampus (McNaughton et al., 1989; Brun et al., 2002). Recent hippocampal place cell studies compared the similarity of CA3 and CA1 firing fields after stimulus changes. Large changes (remapping) were interpreted as pattern separation and stability as pattern completion. CA1 fields changed more than CA3 fields in the study by Lee et al. (2004), but in the study by Leutgeb et al. (2004), changes were greater in CA3 than CA1. The data suggest pattern separation and completion are neither exclusive nor fixed properties of CA3 and CA1. These studies and a third (Vazdarjanova and Guzowski, 2004) suggest there is a nonlinear, dynamic tension in CA3, which maintains CA3 responses to perturbations of familiar stimuli and changes responses to novel environmental geometry. CA1, on the other hand, has a more linear response to stimulus manipulations that varies continu- 
ously between separation and completion (Guzowski et al., 2004).

The present data complement the results from the studies of hippocampal cellular behavior reviewed above. The pair of opposite dissociations indicate the ability to segregate spatial stimuli for organizing behavior and the ability to represent spatial stimuli for organizing behavior are also dissociable hippocampusdependent properties. It is natural to assume that behavioral (perceptual) segregation depends on neural stimulus separation, whereas a reliable place response depends on a maintained neural representation of the relevant associations that were acquired by experience. Another behavioral example is the demonstration that dentate gyrus lesions impaired discrimination of spatial but not temporal separation, whereas CA1 lesions impaired discrimination of temporal but not spatial separation (Gilbert et al., 2001). The distinction between segregating and associating hippocampal operations seems quantitative, suggesting attempts to characterize quantitative functional differentiations may be more productive than attempts to localize functions (Barnes et al., 1990).

\section{Segregation and representation-distinct hippocampal functions}

The present data support the notion that the hippocampus performs at least two functions. First, in accord with associative memory hypotheses, the hippocampus encodes associative representations in a distributed network to support spatial behavior. Because place navigation to a small target in a large environment requires a fine representation of the environment, particularly near the target (Hollup et al., 2001), water maze escape is likely to place a greater demand on this representational function, whereas place avoidance may only require a relatively coarse spatial representation of the large to-be-avoided region (Fig. 1A).

The Room + avoidance task challenged the rat to separate relevant stationary room stimuli from irrelevant self-motion stimuli and presented a relatively mild challenge to represent the location of shock. The responses of hippocampal place cells to dissociating room and self-motion stimuli (Gothard et al., 1996; Redish et al., 2000; Rosenzweig et al., 2003) provide direct evidence of this dynamic segregation in hippocampal discharge. During the TTX blockade, the remaining associative distributed network could operate on the already segregated room inputs to retrieve a relatively coarse spatial representation that was sufficient to support place avoidance.

The TTX injection did not impair learning a Room + Arena + avoidance on a stationary arena when both room and arena cues were relevant to the task (Wesierska et al., 2005), because according to the hypothesis (Fig. $1 C$ ), this task presents no explicit segregation demand and only a modest representational demand. Although Room + Arena - avoidance (Cimadevilla et al., 2000) on a dry rotating arena has the same modest representational demand as the Room + and Room + Arena + task variants, the Room + Arena - task challenges the rat to do stimulus segregation even more than the Room + task. Room + Arena - avoidance requires the segregation of relevant stationary room cues from irrelevant rotating olfactory arena stimuli and self-motion (Fig. 1C). In line with the hypothesis (Fig. 1), the unilateral TTX injection prevented both learning (Wesierska et al., 2005) and retrieval, and even consolidation (Cimadevilla et al., 2001), of Room + Arena - avoidance. According to the hypothesis, the segregation demand is too great in Room + Arena - avoidance to tolerate even mild hippocampal dysfunction (Fig. 1C). Note, however, that there is an additional challenge in Room + Arena- avoidance and even more so in the Room + Arena + (Fenton et al., 1998) avoidance task in which the rat must avoid both a stationary and a rotating place, because entering either region is punished by footshock. The rat must segregate stationary room and rotating arena stimuli and selectively associate shock with the appropriate stimulus subset. This hippocampus-dependent "subgrouping" of relevant associated stimuli (Wesierska et al., 2005) suggests that hippocampal activity is dynamically grouped into active and suppressed representations, a process called cognitive coordination (Phillips and Silverstein, 2003).

\section{Is segregation inherent or learned?}

Substantial work indicates hippocampal representations are learned, but is stimulus segregation also learned? Place preference navigation (Rossier et al., 2000) training on the rotating arena increased the prevalence of room frame-specific and conjunctive room and arena frame-specific place cell discharge patterns in response to continuous rotation. Disorganized responses were reduced by navigation training, and this acquired "frame specificity" was preserved when the rats were no longer navigating (Zinyuk et al., 2000; Fenton et al., 2002), suggesting that at the level of cellular behavior, the separate representation of room and arena information is acquired during place learning on a rotating arena. Similarly, Bostock et al. (1991), Lever et al. (2002), and Hayman et al. (2003) demonstrated that place cell remapping developed with experience, indicating neural stimulus separation is also an acquired property.

If segregating conflicting room and arena inputs occurred early in hippocampal processing and this was instantiated by tuning synapses to realize a separating filter, then the residual associative network properties assumed to underlie the associative functions of the hippocampus could operate on the segregated input patterns. This segregation is critical on the rotating arena in the Room + , Room + Arena -, and Room+Arena + avoidance tasks in which subsets of stimuli are dissociated continuously. Impairment of this segregation would abolish subgrouping, causing behavioral disorganization and impaired selection of appropriate behaviors (Gray and McNaughton, 2000; McNaughton and Wickens, 2003) that might manifest as retarded learning despite an intact ability to form associations. This viewpoint may account for the slower water maze escape after the TTX injections in experiment 2, whereas the spared localized search in the same experiment would reflect sparing of the representational function.

\section{References}

Barnes CA, McNaughton BL, Mizumori SJY, Leonard BW, Lin L-H (1990) Comparison of spatial and temporal characteristics of neuronal activity in sequential stages of hippocampal processing. In: Understanding the brain through the hippocampus (Storm-Mathisen J, Zimmer J, Ottersen OP, eds), pp 287-300. Amsterdam: Elsevier.

Bostock E, Muller RU, Kubie JL (1991) Experience-dependent modifications of hippocampal place cell firing. Hippocampus 1:193-206.

Brun VH, Otnass MK, Molden S, Steffenach HA, Witter MP, Moser MB, Moser EI (2002) Place cells and place recognition maintained by direct entorhinal-hippocampal circuitry. Science 296:2243-2246.

Bureš J, Burešova O (1990) Reversible lesions allow reinterpretation of system level studies of brain mechanisms of memory. Concepts Neurosci $1: 69-89$.

Bureš J, Fenton AA, Kaminsky Y, Rossier J, Sachetti B, Zinyuk L (1997) Dissociation of exteroceptive and idiothetic orientation cues: effect on hippocampal place cells and place navigation. Proc R Soc Lond B Biol Sci 352:1515-1524.

Cimadevilla JM, Kaminsky Y, Fenton AA, Bureš J (2000) Passive and active avoidance as a tool of spatial memory research in rats. J Neurosci Methods 102:155-164. 
Cimadevilla JM, Wesierska M, Fenton AA, Bureš J (2001) Inactivating one hippocampus impairs avoidance of a stable room-defined place during dissociation of arena cues from room cues by rotation of the arena. Proc Natl Acad Sci USA 98:3531-3536.

Fenton AA, Bureš J (1993) Place navigation in rats with unilateral tetrodotoxin inactivation of the dorsal hippocampus: place but not procedural learning can be lateralized to one hippocampus. Behav Neurosci 107:552-564.

Fenton AA, Bureš J (1994) Interhippocampal transfer of place navigation monocularly acquired by rats during unilateral functional ablation of the dorsal hippocampus and visual cortex by lidocaine. Neuroscience 58:481-491.

Fenton AA, Arolfo MP, Nerad L, Bureš J (1995) Interhippocampal synthesis of lateralized place navigation engrams. Hippocampus 5:16-24.

Fenton AA, Wesierska M, Kaminsky Yu, Bureš J (1998) Both here and there: simultaneous expression of autonomous spatial memories in rats. Proc Natl Acad Sci USA 95:11493-11498.

Fenton AA, Bureš J, Cimadevilla JM, Olypher AV, Wesierska M, Zinyuk L (2002) Place cell activity during overtly purposeful behavior (in dissociated reference frames). In: The neural basis of navigation: evidence from single cell recording (Sharp PE, ed), pp 59-80. New York: Kluwer.

Gilbert PE, Kesner RP, Lee I (2001) Dissociating hippocampal subregions: a double dissociation between dentate gyrus and CA1. Hippocampus 11:626-636.

Gothard KM, Skaggs WE, McNaughton BL (1996) Dynamics of mismatch correction in the hippocampal ensemble code for space: interaction between path integration and environmental cues. J Neurosci 16:8027-8040.

Gray JA, McNaughton N (2000) The neuropsychology of anxiety: an enquiry into the functions of the septo-hippocampal system. Oxford: Oxford UP.

Guzowski JF, Knierim JJ, Moser EI (2004) Ensemble dynamics of hippocampal regions CA3 and CA1. Neuron 44:581-584.

Harlan RE, Shivers BD, Kow LM, Pfaff DW (1983) Estrogenic maintenance of lordotic responsiveness: requirement for hypothalamic action potentials. Brain Res 268:67-78.

Hayman RM, Chakraborty S, Anderson MI, Jeffery KJ (2003) Contextspecific acquisition of location discrimination by hippocampal place cells. Eur J Neurosci 18:2825-2834.

Hollup SA, Molden S, Donnett JG, Moser MB, Moser EI (2001) Accumulation of hippocampal place fields at the goal location in an annular watermaze task. J Neurosci 21:1635-1644.

Ježek K, Wesierska M, Fenton AA (2002) Hippocampus-dependent retrieval and hippocampus-independent extinction of place avoidance navigation, and stress-induced out-of-context activation of a memory revealed by reversible lesion experiments in rats. Physiol Res 51 [Suppl 1]:S35-S47.

Kesner RP, Gilbert PE, Wallenstein GV (2000) Testing neural network models of memory with behavioral experiments. Curr Opin Neurobiol 10:260-265.

Klement D, Pǎst'alková E, Fenton AA (2005) Tetrodotoxin infusions into the dorsal hippocampus block non-locomotor place recognition. Hippocampus 15:460-471.

Lee I, Yoganarasimha D, Rao G, Knierim JJ (2004) Comparison of population coherence of place cells in hippocampal subfields CA1 and CA3. Nature 430:456-459.

Leutgeb S, Leutgeb JK, Treves A, Moser MB, Moser EI (2004) Distinct ensemble codes in hippocampal areas CA3 and CA1. Science 305:12951298.

Lever C, Wills T, Caccuci F, Burgess N, O’Keffe J (2002) Long-term plastic- ity in hippocampal place cells representation of environmental geometry. Nature 416:90-94.

Lisman JE (1999) Relating hippocampal circuitry to function: recall of memory sequences by reciprocal dentate-CA3 interactions. Neuron 22:233-242.

Marr D (1971) Simple memory: a theory for archicortex. Philos Trans R Soc Lond B Biol Sci 262:23-81.

McClelland JL, McNaughton BL, O'Reilly RC (1995) Why there are complementary learning systems in the hippocampus and neocortex: insight from the successes and failures of connectionist models of learning and memory. Psychol Rev 102:419-457.

McNaughton BL, Morris RGM (1987) Hippocampal enhancement and information storage within a distributed memory system. Trends Neurosci 10:408-415.

McNaughton BL, Nadel L (1989) Hebb-Marr networks and the neurobiological representation of action in space. In: Neuroscience and connectionist theory (Gluck MA, Rumelhart DE, eds), pp 1-63. Hillsdale, NJ: Erlbaum.

McNaughton N, Wickens J (2003) Hebb, pandemonium and catastrophic hypermnesia: the hippocampus as a suppressor of inappropriate associations. Cortex 39:1139-1163.

McNaughton BL, Barnes CA, Meltzer J, Sutherland RJ (1989) Hippocampal granule cells are necessary for normal spatial learning but not for spatially-selective pyramidal cell discharge. Exp Brain Res 76:485-496.

Moser EI, Moser MB (1998) Distributed encoding and retrieval of spatial memory in the hippocampus. J Neurosci 18:7535-7542.

O'Reilly RC, McClelland JL (1994) Hippocampal conjunctive encoding, storage, and recall: avoiding a trade-off. Hippocampus 4:661-682.

Phillips WA, Silverstein SM (2003) Convergence of biological and psychological perspectives on cognitive coordination in schizophrenia. Behav Brain Sci 26:65-82.

Redish AD, Rosenzweig ES, Bohanick JD, McNaughton BL, Barnes CA (2000) Dynamics of hippocampal ensemble activity realignment: time versus space. J Neurosci 20:9298-9309.

Rolls ET, Treves A (1998) Neural networks and brain function. Oxford: Oxford UP.

Rosenzweig ES, Redish AD, McNaughton BL, Barnes CA (2003) Hippocampal map realignment and spatial learning. Nat Neurosci 6:609-615.

Rossier J, Kaminsky Y, Schenk F, Bureš J (2000) The place preference task, a task allowing new perspectives in studying the relation between behavior and place cell activity in rats. Behav Neurosci 14:273-284.

Shapiro ML, Olton DS (1994) Hippocampal function and interference. In: Memory systems (Schacter DL, Tulving E, eds), pp141-146. London: MIT.

Vazdarjanova A, Guzowski JF (2004) Differences in hippocampal neuronal population responses to modifications of an environmental context: evidence for distinct, yet complementary, functions of CA3 and CA1 ensembles. J Neurosci 24:6489-6496.

Wesierska M, Dockery C, Fenton AA (2005) Beyond memory, navigation and inhibition: behavioural evidence for hippocampus-dependent cognitive coordination in the rat. J Neurosci 25:2413-2419.

Zhuravin IA, Bureš J (1991) Extent of the tetrodotoxin induced blockade examined by pupillary paralysis elicited by intracerebral injection of the drug. Exp Brain Res 83:687-690.

Zinyuk L, Kubík S, Kaminsky Y, Fenton AA, Bureš J (2000) Understanding hippocampal activity by using purposeful behavior: place navigation induces place cell discharge in both task-relevant and task irrelevant spatial reference frames. Proc Natl Acad Sci USA 97:3771-3776. 